\title{
Common extensor tendon rupture following corticosteroid injection for lateral tendinosis of the elbow
}

\author{
Andrew G Smith, Kornad Kosygan, Hywel Williams, Raymond J Newman
}

\begin{abstract}
Corticosteroid injections are commonly administered to athletes to relieve symptoms of lateral elbow tendinosis. This report presents a case of almost total rupture of the common extensor origin in a 45 year old female squash player secondary to such a procedure.

(Br F Sports Med 1999;33:423-425)
\end{abstract}

Keywords: corticosteroid injection; elbow; tendinosis; tendon rupture; squash; tennis elbow

Previous reports have highlighted the possible complication of tendon rupture associated with corticosteroid injections for inflammatory or traumatic conditions of the flexor digital, ${ }^{1}$ patellar, ${ }^{2}$ and Achilles ${ }^{3}$ tendons, as well as the rotator cuff, plantar fascia, ${ }^{56}$ and elsewhere. ${ }^{7}$ We report here a case of corticosteroid injection for lateral tendinosis of the elbow (lateral epicondylitis, lateral tendinopathy, tennis elbow ${ }^{8}$ ) in a squash player resulting in rupture of the common extensor tendons.

\section{Case report}

A 45 year old female high level squash player presented to her general practitioner with signs and symptoms of lateral tendinosis of her dominant elbow. She was treated with an injection of triamcinolone, $40 \mathrm{mg}$ in $1 \mathrm{ml}$, which was particularly painful both during and after the injection. The outer aspect of the elbow became swollen and painful but this subsided. After this, the patient noted a large defect over the outer aspect of the elbow with pallor of the skin. This was associated with pain and weakness of the elbow resulting in inability to play squash.

The patient underwent extensive physiotherapy with little benefit. The skin discoloration improved but the patient was left with a deep defect over the common origin of the extensor tendons and episodes of sharp pain down her arm mainly over the radial aspect. Examination disclosed an obvious defect in the origin of the common extensor tendons, with no more than small palpable anterior and posterior bands remaining. The defect was sufficiently large to allow the easy placement of the tip of the examiner's index finger (fig 1).

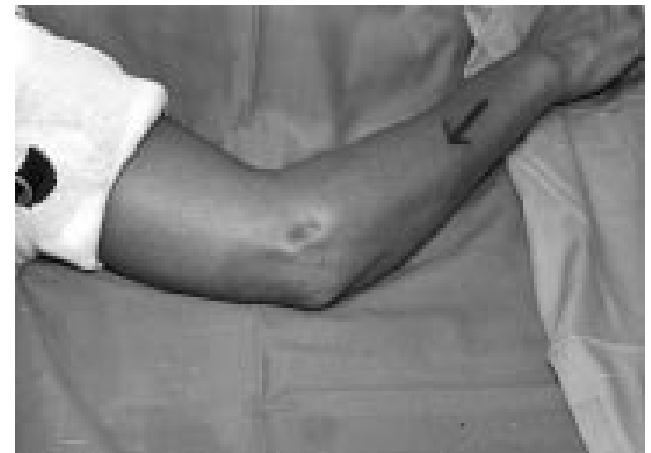

Figure 1 Preoperative photograph of the elbow with visible defect in the origin of the common extensor tendons.

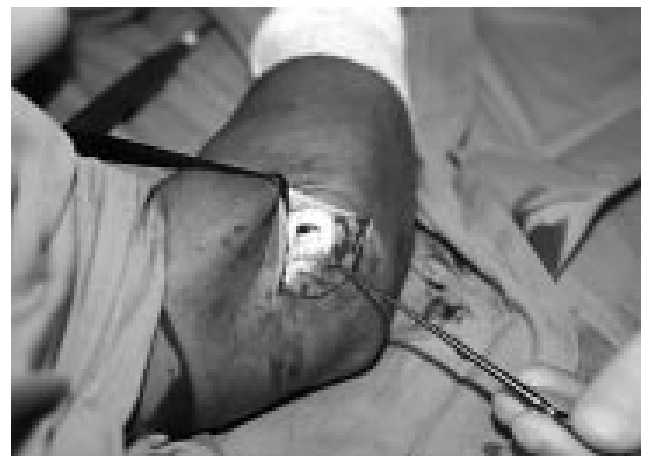

Figure 2 Intraoperative photograph of the elbow showing the rupture in the common extensor tendons close to the lateral epicondyle.

Radiographs showed no bony abnormality, and nerve conduction studies excluded a concomitant nerve injury. At surgery, rupture of the central three quarters of the origin of the common extensor tendons was found (fig 2).

The margins of the dehiscence were excised and the common extensors released from the anterior and posterior aspects of the lateral supracondylar ridge allowing a primary side to side repair of the tendon.

Histological examination of the tendon origin showed fibrillary degeneration of collagenous fibrous tissue, fibrinous exudate, minimal organisation, and no regenerative activity. Fibrillary change continued into the area of blending of tendon and skeletal muscle. Skeletal muscle fibres showed intense regenerative activity with areas of fibroblastic
Accepted for publication 15 June 1999 
scarring but no myocytic necrosis. Inflammation was completely lacking in the tendon.

\section{Discussion}

The literature indicates that, if corticosteroid injections are placed within tendons, they may be associated with a deleterious effect on the tendon tissue. ${ }^{9}$ In this case, three processes were identified histologically: noninflammatory degeneration of collagen, separation of the myotendinous junction, and synovial fibrinous exudate. It would therefore appear that the corticosteroid injected caused collagen degeneration which had become fibrillary, permitting a traction injury to occur at the myotendinous junction. The absence of a regenerative response may have predisposed to rupture. Nearby muscle showed brisk regeneration with some fibrosis and scarring. Similar effects have been confirmed in animal studies. ${ }^{3}$

In clinical practice, corticosteroid injections are widely and generally successfully used for treating lateral tendinosis in athletes both in general practice and the hospital setting. In general terms, the use of corticosteroid injections is safe and has a low complication rate when performed with adequate precautions. ${ }^{10}$ Massive rupture of the common extensor origin is an uncommon complication but one with devastating effects, particularly in an athletic person who participates in racket sports.
This case report underlines the need to take care in placing injections sufficiently deep to avoid subcutaneous lipoatrophy, ${ }^{10}{ }^{11}$ but not so deep as to cause intratendinous placement. A practical tip may be to withdraw the needle a little if an unusual amount of resistance is encountered at the start of the injection.

We wish to thank Dr C Gray, consultant pathologist, for assistance in interpreting the histology results.

1 Gottlieb NL, Riskin WG. Complications of local corticosteroid injections. FAMA 1980;243:1547-8.

2 Morgan J, McCarty DJ. Tendon ruptures in patients with systemic lupus erythematosus treated with corticosteroids. Arthritis Rheum 1974;17:1033-6.

3 Shrier I, Gordon O. Achilles tendon: are corticosteroid injections useful or harmful? Clin ₹ Sport Med 1996;6:24550 .

4 Mahler F, Fritsch YD. Partial and complete ruptures of the Achilles tendon and local corticosteroid injections. $\mathrm{Br} F$ Sports Med 1992;26:7-14.

5 Acevedo JI, Beskin JL. Complications of plantar fascia rupture associated with corticosteroid injection. Foot Ankle Int 1998:19:91-7.

6 Sellman JR. Plantar fascia rupture associated with corticosteroid injection. Foot Ankle Int 1994;15:376-81.

7 Ford LT, DeBender J. Tendon rupture after local steroid injection. South Med $\mathcal{f}$ 1979;72:827-30.

8 Maffulli N, Khan KM, Puddu G. Overuse tendon conditions. Time to change a confusing terminology. Arthroscopy 1988;14:840-3.

9 Fredberg U. Local corticosteroid injection in sport: review of literature and guidelines for treatment. Scand $7 \mathrm{Med} S \mathrm{Sc} i$ Sport 1997;7:131-9.

10 Kumar N, Newman RJ. Complications of intra- and peri-articular steroid injections. Br f Gen Pract 1999;49: 465-6.

11 Newman RJ. Local skin depigmentation due to corticosteroid injections. BMF 1984;288:1725-6.

Take home message

(a) Significant complications may be associated with the use of corticosteroid injections for lateral tendinosis of the elbow. (b) Rupture of the common extensor origin can occur. (c) Practical tip: withdraw the needle slightly if an unusual amount of resistance is encountered on injecting the material.

\section{Commentary}

To inject or not to inject corticosteroid remains the question. The few randomised placebo controlled trials of corticosteroid injection in tendinopathy provide conflicting results as to long term outcome, ${ }^{1-3}$ while reporting short term benefit. ${ }^{1}$ Clinical experience suggests that the patient sometimes benefits, and thus injection can seem attractive. Randomised trials of corticosteroid efficacy are needed, as the treatment is not without risk, as shown in this case report.

In this case, the injection may have been intratendinous. Animal experiments prove that intratendinous injection causes collagen necrosis ${ }^{4}$ and decreased load to failure. There is no evidence that paratendinous injection is similarly deleterious. ${ }^{5}$ As tendon rupture can occur whether or not corticosteroid is injected, this case is a salutary reminder to any clinician considering injecting corticosteroid that the possibility of rupture must be raised with the patient, and the discussion noted. Some lawyers understand neither pathophysiology of tendon rupture nor statistics. On balance, if the patient and doctor remain uncertain as to whether or not to proceed with injection, it may be as well to heed Hippocrates' advice: Primum non nocere.

KARIM KHAN

Vancouver

1 Stahl S, Kaufman T. The efficacy of an injection of steroids for medial epicondylitis. A prospective study of sixty elbows. $\mathcal{F}$ Bone foint Surg [Am] 1997;79:1648-52.

2 Day BH, Govindasamy N, Patnaik R. Corticosteroid injections in the treatment of tennis elbow. The Practitioner 1978;220:45962.

3 DaCruz DJ, Gleeson M, Allen MJ, et al. Achilles paratenonitis: an evaluation of steroid injection. Br f Sports Med 1988;22:645 .

Balasubramaniam P, Prathap K. The effect of injection of hydrocortisone into rabbit calcaneal tendons. F Bone foint Surg [Br] 1972;54:729-34. 5 Fredberg U. Local corticosteroid injection in sport: a review of literature and guidelines for treatment. Scand F Med Sci Sport 


\section{Commentary}

This paper serves to remind us again of the potential pitfalls of corticosteroid injections. One wonders if the painful nature of the injection in this subject might have indicated that the injection was being administered into the common extensor tendon origin. Doctors administering periarticular injections need to be aware that the subjective feel of the syringe plunger is an important indicator of the resistance against the injection fluid made by the anatomical structures. Invariably a high resistance indicates a "dangerous" injection into an anatomical structure rather than a low resistance indicating a "safe" injection into a tissue space or anatomical plane.

In the current medicolegal climate, best practice should also involve informed consent of the patient who should understand the risks of a corticosteroid injection. This is best achieved by giving them an appropriate information sheet before the injection. The text below is the current corticosteroid injection handout used at the British Olympic Medical Centre, and is reproduced with permission.

\section{Corticosteroid injections}

If you have an inflamed joint, or if you have pain or inflammation near a joint, you may need an injection of corticosteroid preparation into the affected area.

Injecting into a joint is called an "intra-articular" injection. Injecting near a joint but not actually into it is called a "peri-articular" injection (meaning "near the joint") or "soft tissue" injection. Sometimes a local anaesthetic is used as well as the corticosteroid.

WHY DO I REQUIRE A LOCAL STEROID INJECTION?

An intra-articular injection is given to reduce inflammation, swelling, and pain within a joint. A peri-articular injection is given to reduce pain and inflammation around a joint.

HOW QUICKLY WILL THE STEROID INJECTION TAKE TO WORK, AND HOW LONG WILL IT LAST?

This varies between patients, but usually improvement starts in 3-4 days. If it is helpful, the benefit usually lasts from a few weeks to several months. At the same time other factors in your life can be changed to prevent the problem recurring.

ARE THERE ANY SIDE EFFECTS?

Side effects are very unlikely. Very occasionally patients notice a flare in their joint pain within the first 24 hours after an injection. This usually settles spontaneously over the next couple of days. You can use paracetamol if this is uncomfortable.

Very rarely infection might be introduced into the joint at the time of an injection, so if the joint becomes more painful and hot, then you should consult your doctor immediately.

Occasionally with peri-articular injections some thinning and pallor of the skin may occur at the injection site. Very rarely a tendon may rupture as a result of the injection.

DO I NEED TO REST AFTER THE INJECTION?

Yes, you should rest as much as possible for the first 2 days after an intra-articular injection. This is especially important if a weight-bearing joint such as the knee or ankle has been injected.

If you are to have a peri-articular injection you will be advised how long to rest. In most cases the area should be used minimally for a week.

ROD JAQUES

British Olympic Medical Centre, Northwick Park Hospital, London 